\title{
PID and Fuzzy Logic Controller Design for Balancing Robot Stabilization
}

\author{
Firas A. Raheem ${ }^{1}$, Bashar F. Midhat ${ }^{2}$, Hussein S. Mohammed ${ }^{3}$ \\ University of Technology, Control \& Systems Engineering Department, Baghdad, Iraq, \\ 1dr.firas7010@yahoo.com, ${ }^{2} 60170 @ u o t e c h n o l o g y . e d u . i q,{ }^{3}$ hussein.mashaan@gmail.com
}

\begin{abstract}
This paper addresses the problem of position control and stabilization for the two wheeled balancing robot. A mathematical model is derived based on the robot's position and tilt angle and a fuzzy logic control is proposed for the balancing robot control. The fuzzy logic controller performance is compared with a conventional PID controller to show the difference between them. Both controllers were tested on the balancing robot in simulation using MATLAB software and the results were put together for a comparative point of view. The simulations shows a relative advantage for the fuzzy logic controller over the conventional PID controller especially in reducing the time required for stabilization which takes about 2 seconds and almost without overshoot while in the PID case the robot will have about $10 \%$ overshoot in position and about 20 degrees in tilt angle.
\end{abstract}

Index Terms - Balancing Robot, PID controller, Fuzzy logic control, MATLAB.

\section{Introduction:}

A Two wheeled balancing robot is a mechanical system consisting a thin rod attached at its bottom to a moving cart and the whole system (the rod and cart) balances on only two wheels attached to the cart [1].

The balancing robot stabilization is considered as a challenge for control engineering since the robot model involves a highly nonlinear model dynamics and also because of the unstable nature of the balancing robot which means that the force must be applied properly to the two wheels in order to keep the balancing robot in an upward position or other else the balancing robot will be subjected to fall down, thus in order to achieve this, proper controller is required [2].

Over the past few decades, the research on two wheeled mobile robot or commonly known as balancing robot have gained momentum in a number of robotic centers around the world, the reasons of using balancing robots as a transportation vehicle are better maneuvering and turning control in narrow spaces. Various controllers are implemented on two wheeled balancing robot. Due to its simplicity in implementation, one of the first feedback control schemes addressed for the two wheeled robot stabilization was the conventional PID controller. However, the real difficulty when applying the PID controller was presented in choosing the appropriate gains and the performance often was subjected to high overshoots and oscillations [2].

A Proportional Integral Derivative - Active Force Control (PID-ACF) methods to swing a pendulum attached to a cart from an initial downwards position to an upright position and keep that condition stable was simulated in [3] and implemented to the segway chair human transporter. On the other hand, the linear quadratic regulator (LQR) found to be able to stabilize the two-wheeled balancing robot system in a short period of time [4]. The drawback presented when applying the LQR control was that the precise model of the robot should be known and the performance was highly sensitive for the parameter changes such as load variation. Another approach was a blend controller based on states feedback control embedded with the PID speed synchronization for enhancing the robot stability [5]. 
A visual servoing technique was applied in [6] that allows the robot to follow the line on the floor captured by a camera as the desired trajectory.

Over the past few decades the fuzzy logic control (FLC) had emerged and applied to a wide variety of applications. One of the main aspects of the FLC is the high insensitivity to the plant model which makes it favorable to control the nonlinear systems since there's no need to know the precise model of the plant. In [7], a Fuzzy Immune PD Algorithm was researched and addressed for the balancing robot stabilization problem. Another work proposed the Design of Fuzzy Adaptive PID Controller in order to reduce the settling time and large overshoot of Two-Wheeled Self-Balancing Robot [8]. A real-time control platform for the two-wheeled self-balancing robot is designed based on fuzzy PD control algorithm and achieved self-balanced control of the two wheeled robot successfully and prevented the robot from falling while obtaining a good dynamic performance [9].

In this work the mathematical model of the balancing robot is presented then a conventional PID and Fuzzy PID controllers are designed in order to stabilize the robot. After that a simulation is carried where each controller is applied in order to stabilize the robot while moving and results were sketched to show the difference in performance between the two controllers.

\section{Mathematical Model:}

The two-wheeled balancing robot consists of a long body with two wheels mounted at one end (figure (1)). For the simplicity of this derivation, the two wheels will be treated as a unit, and it will be assumed that the robot travels only in a straight line. By properly designing the hardware, modeling can be simplified. Thus the body can be treated as a point mass rotating about the axis of the wheels.

A set of assumptions made to simplify modeling is as follow:

i- The wheels are always in contact with the floor and experience rolling with no slip.

ii- The electrical system response is significantly faster than that of mechanical system, so that the dynamics of the electrical system may be neglected.

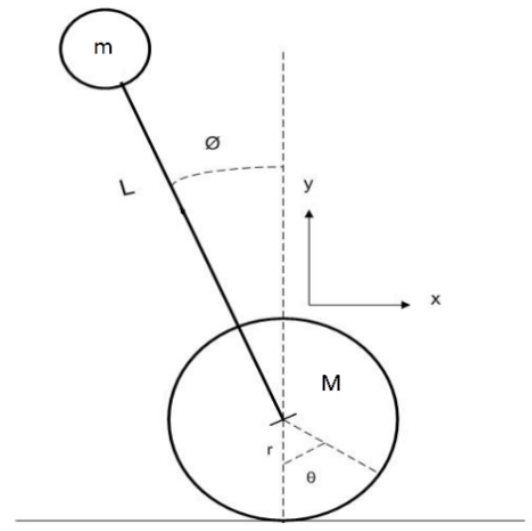

FIGURE (1): SIMPLIFIED DIAGRAM FOR THE BALANCING ROBOT [3]

iii- The motion of the robot is constrained to a straight line, so that the system may be analyzed as 2diminsional system with only planar motion.

iv- All bodies are rigid. 
Considering the above assumptions, the system can be described by two decoupled equations for the translational motion $x$ and rotational motion $\emptyset$ where the translational motion $x$ is substituted for $x=r \theta$ as follows [10].

$$
\begin{aligned}
& \ddot{\mathrm{x}}=\frac{L \cos \emptyset}{0.5 \mathrm{~L}^{2} \mathrm{M}+\mathrm{I}+\mathrm{L}^{2} \mathrm{~m}-0.5 \mathrm{ML}^{2}(\cos \varnothing)^{2}}\left[-\mathrm{T}-\frac{\mathrm{TL}}{\mathrm{r} \cos \emptyset}-\frac{0.5 \mathrm{ML}^{2} \dot{\phi}^{2} \sin \emptyset}{\cos \emptyset}+\frac{\mathrm{Lb}}{\mathrm{r} \cos \emptyset}\left(\frac{\dot{\mathrm{x}}}{\mathrm{r}}-\emptyset\right)+\mathrm{MgL} \sin \emptyset+\right. \\
& \left.\mathrm{b}\left(\frac{\dot{\mathrm{x}}}{\mathrm{r}}-\dot{\emptyset}\right)\right]
\end{aligned}
$$

And

$$
\begin{aligned}
& \ddot{\emptyset}=\frac{1}{0.5 \mathrm{MrL} \cos \varnothing}\left[\mathrm{T}+0.5 \mathrm{Mr}\left[\frac { L \operatorname { c o s } \emptyset } { 0 . 5 \mathrm { L } ^ { 2 } \mathrm { M } + \mathrm { I } + \mathrm { L } ^ { 2 } \mathrm { m } - 0 . 5 \mathrm { ML } ^ { 2 } ( \operatorname { c o s } \varnothing ) ^ { 2 } } \left[-\mathrm{T}-\frac{\mathrm{TL}}{\mathrm{r} \cos \emptyset}-\frac{0.5 \mathrm{ML}^{2} \dot{\phi}^{2} \sin \emptyset}{\cos \emptyset}+\frac{\mathrm{Lb}}{\mathrm{r} \cos \varnothing}\left(\frac{\dot{\mathrm{x}}}{\mathrm{r}}-\right.\right.\right.\right. \\
& \left.\left.\left.\emptyset)+\mathrm{MgL} \sin \emptyset+\mathrm{b}\left(\frac{\dot{\mathrm{x}}}{\mathrm{r}}-\dot{\emptyset}\right)\right]\right]+0.5 \mathrm{Mr} \dot{\emptyset}^{2} \mathrm{~L} \sin \emptyset+\mathrm{rm} \ddot{\mathrm{x}}-\mathrm{b}\left(\frac{\dot{\mathrm{x}}}{\mathrm{r}}-\dot{\emptyset}\right)\right]
\end{aligned}
$$

Where the balancing robot parameters are defined in table (1) below:

TABLE (1): TWO WHEELED BALANCING ROBOT PARAMETERS.

\begin{tabular}{ccc}
\hline Symbol & Parameter & Units \\
\hline$\varnothing$ & Angular Position of Body & $\mathrm{rad}$ \\
$\mathrm{x}$ & Robot position & $\mathrm{m}$ \\
$\mathrm{M}$ & Mass of Body & $\mathrm{kg}$ \\
$\mathrm{L}$ & Length of the Body & $\mathrm{rad}$ \\
$\mathrm{T}$ & Motor torque & $\mathrm{Nm}$ \\
$\mathrm{g}$ & Gravitational acceleration & $\mathrm{m} / \mathrm{sec}^{2}$ \\
$\mathrm{I}$ & Rod moment of inertia & $\mathrm{Kgm}$ \\
$\mathrm{m}$ & End point mass & $\mathrm{kg}$ \\
$r$ & Radius of The Wheel & $\mathrm{m}$ \\
\hline
\end{tabular}

\section{Controller Design}

\section{A. PID Control Design}

The PID controller is considered as one of the most popular control schemes. The main reason for that is the simplicity in implementation. Because that, they are applied to a wide range of applications.

The PID controller can be expressed in Eq. (3) below

$u(t)=K_{p}\left(e(t)+\frac{1}{T_{i}} \int e(t) d t+T_{d} \dot{e}(t)\right)$

Where $u(t)$ is the control action, $e(t)$ is the error and $\dot{e}(t)$ is the error rate of change.

The PID controller constants $\left(K_{p}, T_{i}\right.$ and $\left.T_{d}\right)$ are described as follows:

i) $K_{p}$ is the proportional gain and provides a control action proportional to the error signal e(t).

ii) $T_{i}$ is the integral term. The effect of the integral term in the PID controller is to reduce the steady state error by continuous integration of the error signal e $(\mathrm{t})$.

iii) $T_{d}$ is the derivative term. The derivative term in the PID controller provides a control signal proportional to the error rate of change $\dot{e}(t)$ with respect to time which results in output overshoot damping and hence an improved transient response. 
One point to consider when applying the PID controller is choosing the appropriate values for the gains $K_{p}, T_{i}$ and $T_{d}$. Otherwise, an inappropriate selection for these values may result in worsening the response rather than improving it.

Ziegler and Nichols proposed rules for determining values of the proportional gain $\mathrm{K}_{\mathrm{p}}$, integral gain $K_{i}$, and derivative gain $K_{d}$ based on the transient response characteristics of a given plant [10]. In this method, increase $K_{\mathrm{p}}$ (figure (2)) from 0 to a critical value $K_{\mathrm{cr}}$ at which the output first exhibits sustained oscillations with a period $\mathrm{P}_{\mathrm{cr}}$ (figure (3)), thus, the critical gain $K_{\mathrm{cr}}$ and the corresponding period $\mathrm{P}_{\mathrm{cr}}$ are experimentally determined. Ziegler and Nichols suggested that we set the values of the parameters $K_{p}, T_{i}$, and $T_{d}$ according to table (2) [11]:

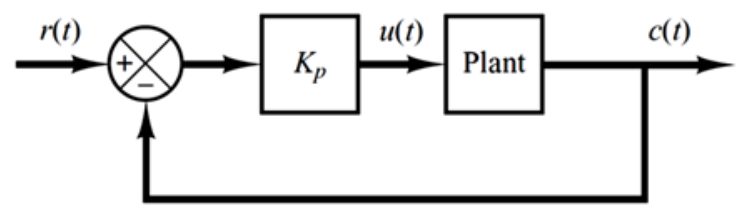

FIGURE (2): CLOSED-LOOP SYSTEM WITH A PROPORTIONAL CONTROLLER.

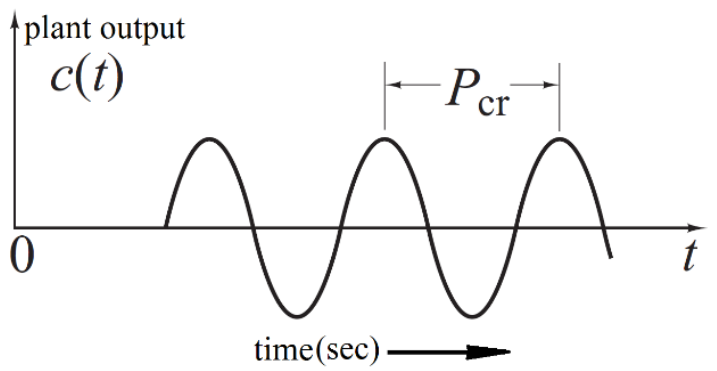

FiguRE (3): SUSTAINED OSCILLATION WITH PERIOD PCR.

Table (2): Ziegler-Nichols Second Method Tuning Rule Based on Critical Gain Kcr and Critical Period Pcr [11].

\begin{tabular}{cccc}
\hline $\begin{array}{c}\text { Type of } \\
\text { Controller }\end{array}$ & $\mathrm{K}_{\mathrm{p}}$ & $\mathrm{T}_{\mathrm{i}}$ & $\mathrm{T}_{\mathrm{d}}$ \\
\hline $\mathrm{P}$ & $0.5 K_{\mathrm{cr}}$ & $\infty$ & 0 \\
$\mathrm{PI}$ & $0.45 K_{\mathrm{cr}}$ & $1.2 \frac{1}{\mathrm{Pcr}}$ & 0 \\
$\mathrm{PID}$ & $0.6 K_{\mathrm{cr}}$ & $0.5 \mathrm{P}_{\mathrm{cr}}$ & $8 \frac{1}{\mathrm{Pcr}}$ \\
\hline
\end{tabular}

\section{B. Fuzzy Logic Control}

Fuzzy logic control (FLC) is a rule-based control method which interprets human experience into reality by utilizing the principles of fuzzy set theory in its data representation. In the last few decades the FLC had attracted the interest of many researchers and was applied to many plants as a novel or successful control approach. The main feature of the FLC is that its structure is independent of the plant model, for this reason it was extensively investigated and applied to systems where the precise mathematical model of the plant was unknown or involves high nonlinearities.

The fuzzy controller is composed of the following three-elements: 
1- Fuzzification step: The input data are converted to linguistic variables which their values determined according to input member ship functions. In this paper the inputs to the FLC will be the error e $(\mathrm{t})$ and its time derivative de/dt. The triangular member ship function was used for input to represent the linguistic variables. The FLC input has five linguistic variables labeled as (Negative big, Negative small, Zero, Positive small, Positive big) and labeled as (NB, NS, Z, PS, PB) respectively.

2- Rule Base: A decision making process simulating a human decision process which includes the definitions of the fuzzy membership functions assigned for each control variable including the definitions of the fuzzy membership functions defined for each control variable and the necessary rules that specify the control goals using linguistic variables.

3- Defuzzification: The inferred decision from the linguistic variables are converted back to numerical values. The input for defuzzification is the member ship (certainty) from implied fuzzy sets resulted from premise rules and the output is a crisp number. Figure (4) shows the general structure of the fuzzy controller including its main components.

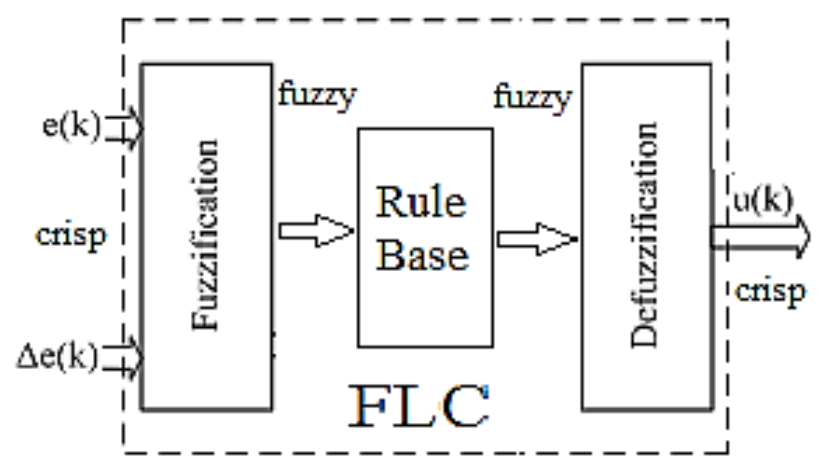

Figure (4): GENERAL STRUCTURE OF THE FUZZY CONTROLLER [4].

The Fuzzy logic controller is designed to stabilize the robot while moving to a specified distance. The inputs to the fuzzy controller are the error value $e$ and the rate of change of the error $\dot{e}$ and one output which is the control action u. Thus the fuzzy logic controller consists of three membership functions; two for the inputs and one for the output as shown below.

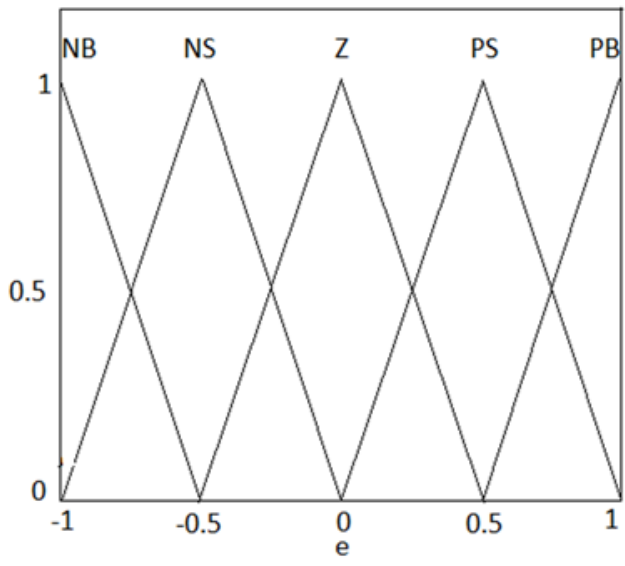

FIGURE (5): MEMBERSHIP FUNCTION OF ERROR E.

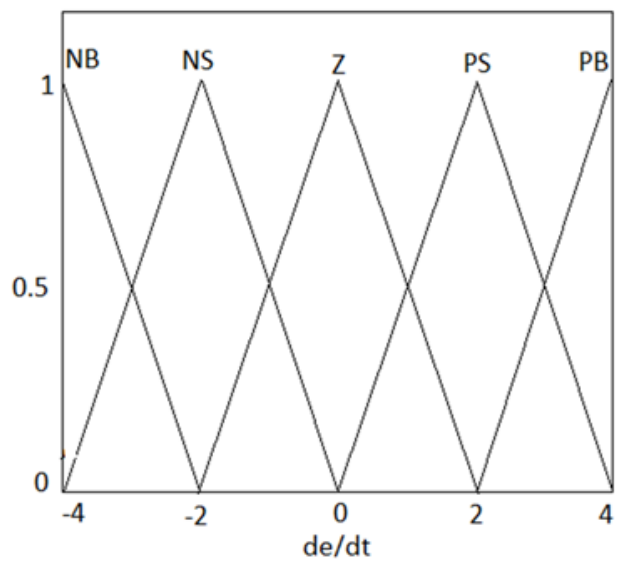

Figure (6): MEMBERSHIP FunCTION OF ERROR RATE OF CHANGE. 


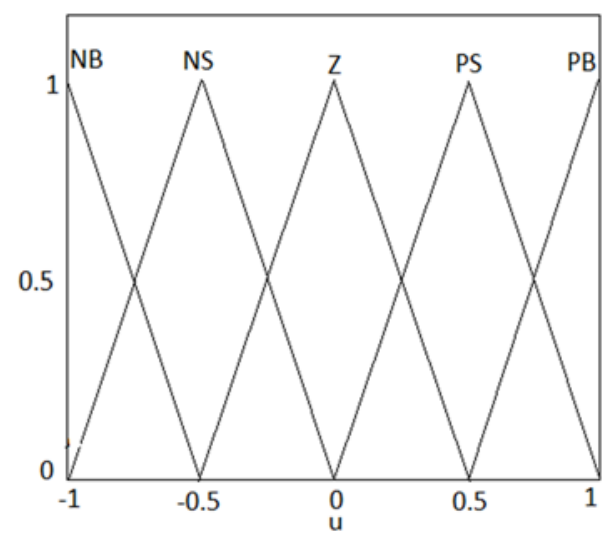

Figure (7): MEMBERSHip FunCtion OF OUTPUT CONTROL U.

On defining the membership functions, fuzzy rule base is formed in a Fuzzy Associative Memory (FAM) table as shown in Table 2. The inference from Table (3) gives the output membership values $u$ of the designed FLC based balancing robot system.

TABLE (3): FUZZY LOGIC CONTROLLER RULE BASE FOR BALANCING ROBOT STABILIZATION.

\begin{tabular}{|c|c|c|c|c|c|}
\hline e & NB & NS & $\mathbf{Z}$ & PS & PB \\
\hline NB & NB & NB & NS & NS & $\mathrm{Z}$ \\
\hline NS & NB & NS & NS & Z & PS \\
\hline $\mathbf{Z}$ & NS & NS & Z & PS & PS \\
\hline PS & NS & Z & PS & PS & PB \\
\hline PB & Z & PS & PS & PB & PB \\
\hline
\end{tabular}

Table (3) indicates the set of 25 rules which makes the given balancing robot system to be stable. Since the balancing robot has two configuration variables; the angle and position, then two controllers are combined to control the robot. The first takes the angle error and tries to stabilize the robot and the other takes the position and drives the robot to the desired location. The control structure is shown in figure (9) below.

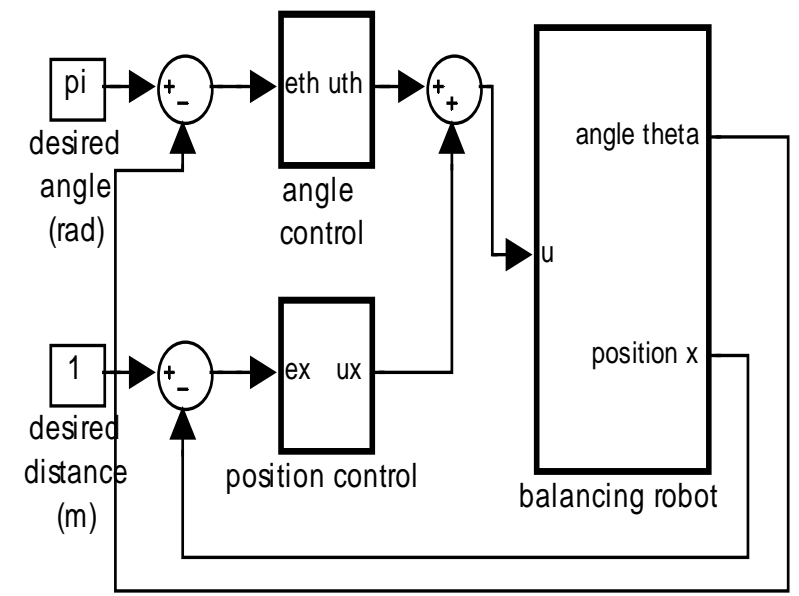

FIGURE (8): CONTROL STRATEGY FOR BOTH POSITION AND ANGLE CONTROL.

\section{Simulation and results}

Simulations were held using MATLAB in order to verify the work done in the previous sections. The simulations were made in three parts to as follows. 


\section{A. Tuning the PID controller}

In this step the Ziegler-Nichols tuning process proposed in section 3.1 was performed in order to find the values of $K_{p}, K_{i}$, and $K_{d}$ of both

First, the PID controller associated with angle control was tuned while the PID controller associated with position control was disabled. $K_{p}$ was increased from 0 to the state where a sustained oscillation of the balancing robot was obtained. The value of critical gain $K_{\text {cr }}$ was found to be 27.26 and the oscillation period was equal to $2.76 \mathrm{sec}$. Applying the values of the critical gain $K_{\mathrm{cr}}$ and the oscillation period in table (2) results in the following values for the PID controller associated with angle control $\left(\mathrm{K}_{\mathrm{pa}}=16.4, \mathrm{~K}_{\mathrm{ia}}=1.34\right.$, and $\left.\mathrm{K}_{\mathrm{da}}=1.5\right)$ where the suffix (a) refers to the PID controller associated with angle control. After tuning the PID controller associated with the balancing robot angle the values obtained for PID gains were applied and the PID controller associated with the robot position was connected and tuned using trial and error process, the tuned PID gains were found as follows for the PID controller associated with position control $\left(\mathrm{K}_{\mathrm{pp}}=-3.2, \mathrm{~K}_{\mathrm{ip}}=-0.26\right.$, and $\left.\mathrm{K}_{\mathrm{dp}}=-2.5\right)$ where the suffix (p) refers to the PID controller associated with position control.

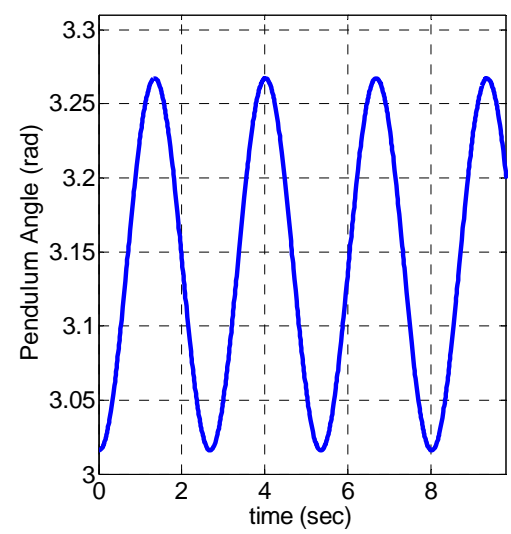

FiguRE (9): BALANCING ROBOT SUSTAINED OSCILLATION.

\section{B. Balancing robot stabilization}

After the tuning process performed to find the appropriate values of the PID controller associated to the angle and position control the tuned controller gains were applied to the balancing robot. Both the PID and fuzzy logic controllers designed in previous sections are applied to the balancing robot described by equations (1) and (2). The simulations were performed using MATLAB Simulink. The robot parameters were taken for an experimental laboratory size balancing robot [7] as in table (4):

TABLE (4): BALANCING ROBOT PARAMETERS VALUES.

\begin{tabular}{cccc}
\hline Parameter & Symbol & Value & Units \\
\hline Mass of main body & $\mathrm{M}$ & 2.4 & $\mathrm{~kg}$ \\
Mass of upper load & $\mathrm{m}$ & 0.23 & $\mathrm{~kg}$ \\
Rod moment of & $\mathrm{I}$ & 0.1 & $\mathrm{kgm}^{2}$ \\
inertia & & & \\
Rod length & $\mathrm{L}$ & 0.4 & $\mathrm{~m}$ \\
Wheel radius & $\mathrm{r}$ & 0.037 & $\mathrm{~m}$ \\
\hline
\end{tabular}

The controller objective is to move the robot to a specified distance ( $1 \mathrm{~m}$ in simulation) while trying to keep the robot in vertical position (i.e. $180 \mathrm{deg}$ ) as much as possible. Figures (10), (11) and (12) show the simulation results. 


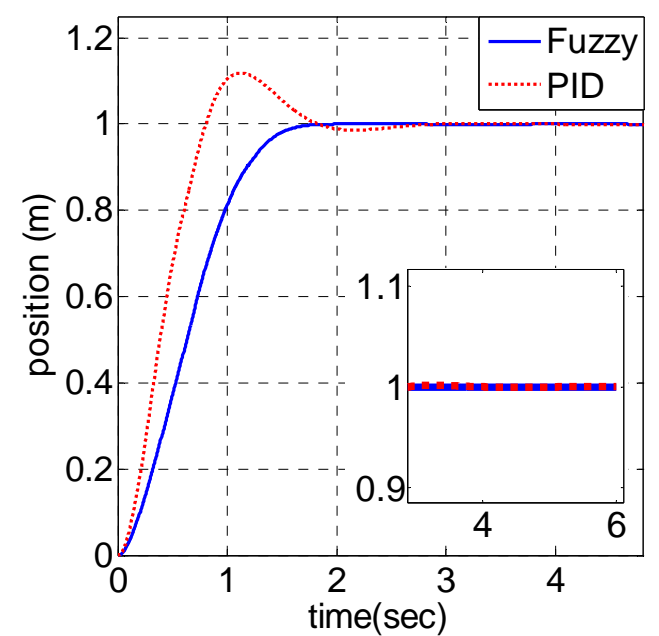

FiguRE (10): ROBOT POSITION VS. TIME.

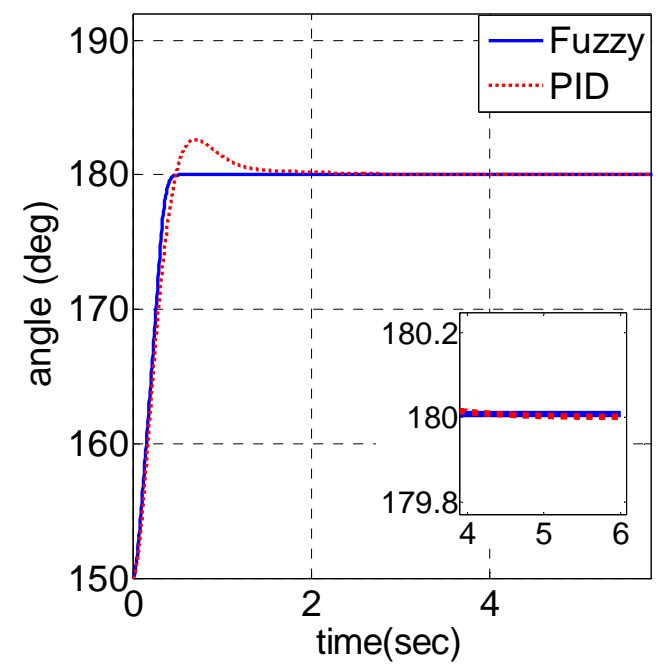

FIGURE (11): RoBOT ANGLE VS. TIME.

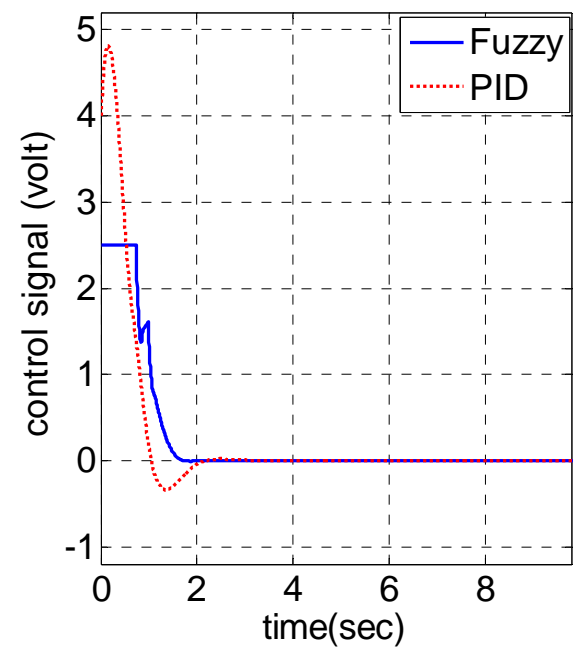

FIGURE (12): CONTROL SIGNAL VS. TIME.

\section{Disturbance rejection}

In this part of simulation, the ability of the proposed fuzzy controller to reject external disturbances was investigated. An external force of about 0.5 Newtons was applied to the balancing robot system for about 0.5 seconds. The simulations results are shown in figures (13), (14) and (15).

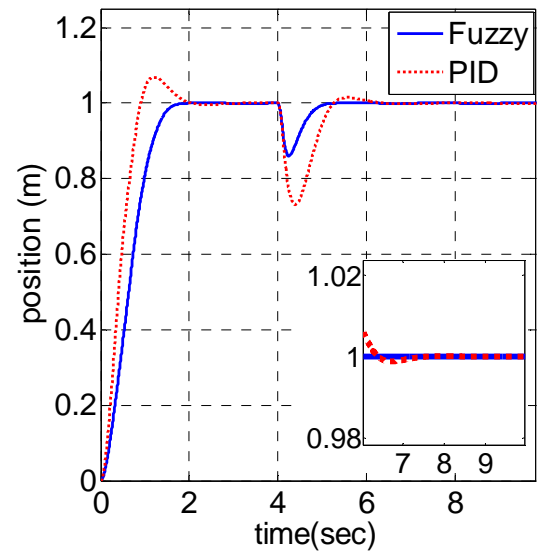

FIGURE (13): ROBOT POSITION VS. TIME.

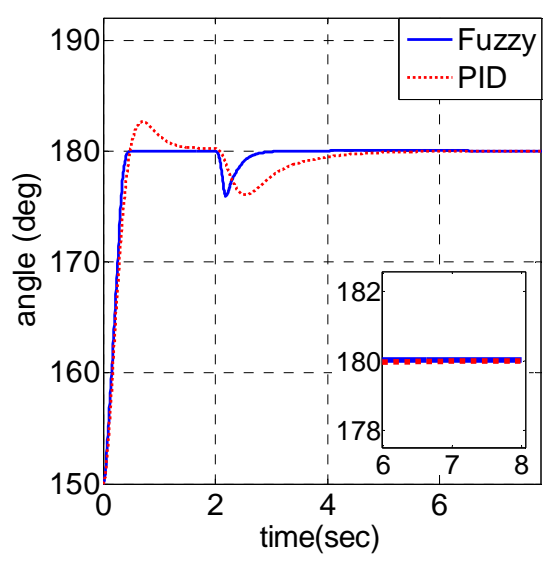

Figure (14): RoBot ANGLE VS. TIME. 


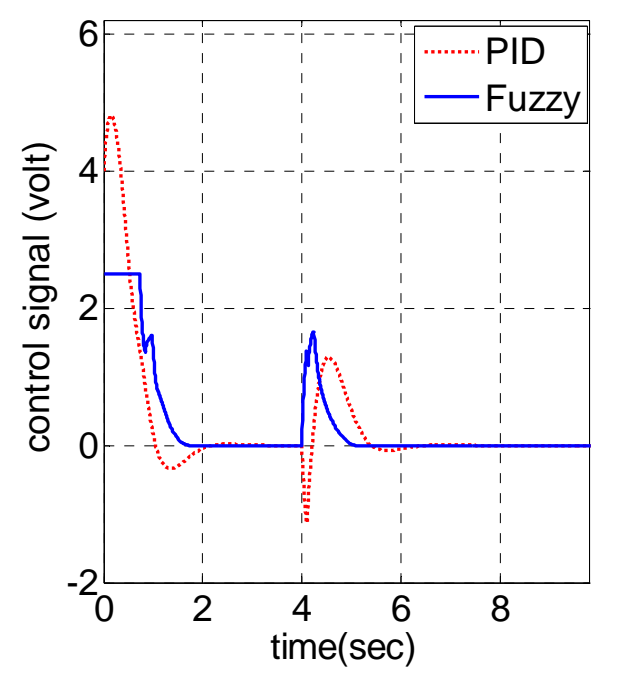

FIGURE (15): CONTROL SIGNAL VS. TIME.

\section{Conclusions}

Two controllers are proposed and designed in this work for the two wheeled balancing robot stabilization problem. Although both controllers was able to stabilize the robot in a finite time, the results show that the fuzzy controller have a better performance indices than the conventional PID controller. First, the proposed fuzzy controller stabilizes the robot within about 2 seconds while the PID will oscillate for about 4 seconds before settling. Also the fuzzy controller has no overshoots in performance while in the PID case the robot will have about $10 \%$ overshoot in position and about 20 degrees in tilt angle. Another point to consider is that the control signal is considerably less in the fuzzy control case which means that the system will consume less energy to move the robot to the specified distance which is preferred for an economical point of view. When the external disturbance was applied to the balancing robot the PID controller was able to stabilize the robot within about 2 seconds while the fuzzy controller was able to stabilize the robot within about 1 second. In addition, when applying the external disturbance, the max position error was about 0.2 meters when using the PID controller to stabilize the balancing robot while when using the fuzzy controller the max position error was less than 0.1 meters which means that the fuzzy controller is more robust than the PID controller.

\section{REFERENCES}

[1] Nor Maniha Abdul Ghani, Faradila Naim, Tan Piow Yon,” Two Wheels Balancing Robot with Line Following Capability”, World Academy of Science, Engineering and Technology, Vol. 55, pp. 634-638, 2011.

[2] Braunl and J. Pan, "Balancing a Two-Wheeled Autonomous Robot”, Electronic Design Engineering, vol. 9, no. 11, (2007), pp.103-106.

[3] Kamajaya Putra, Endra Pitowarno,” Inverted Pendulum Human Transporter Balance Control System Based on Proportional Integral Derivative - Active Force Control”, Electronic Engineering and Polytechnic Journal, Vol. 11, No. 6, pp.139-144, 2016.

[4] L. Yang and L. YI-we, "Simulation Research on Self-balanced Two-wheel Vehicle Control Algorithm Based on PID and LQR”, MECHANICAL ENGINEERING \& AUTOMATION, pp.7-10, 2011.

[5] Li Chaoquan, Gao Xueshan and Li Kejie, "Smooth Control the Coaxial Self-Balance Robot Under Impact Disturbances”, International Journal of Advanced Robotic Systems, Vol. 8, No. 2, pp. 59-67, 2016.

[6] Jian Fang,’The research on the Application of Fuzzy Immune PD Algorithm in the Two-Wheeled and Self-Balancing Robot System”, International Journal of Control and Automation Vol.7, No.10 (2014), pp.109-118.

[7] Congying Qiu and Yibin Huang,’The Design of Fuzzy Adaptive PID Controller of Two-Wheeled Self-Balancing Robot”, International Journal of Information and Electronics Engineering, Vol. 5, No. 3, pp. 193-197, May 2015.

[8] G. H. Lee1 and Seul Jung1,'Line Tracking Control of a Two-Wheeled Mobile Robot Using Visual Feedback”, International Journal of Advanced Robotic Systems, Vol. 9, No. 1, pp. 177 -185, 2017. 
[9] Chaoquan Li and Xueshan Gao, Qiang Huang, “A coaxial couple wheeled robot with T-S fuzzy equilibrium control”, Industrial Robot Journal, Vol. 38, No. 3, pp. 292-300, 2011.

[10] Amir A. Bature1, Salinda Buyamin, Mohamed. N. Ahmad and Mustapha Muhammad, “ A Comparison of Controllers for Balancing Two Wheeled Inverted Pendulum Robot”, International Journal of Mechanical \& Mechatronics Engineering, Vol. 14, No. 03, pp.62-68, 2014.

[11] Katsuhiko Ogata, “Modern Control Engineering”, Fifth Edition, Prentice Hall, 2010. 